\title{
Formation of offshore tidal sand banks triggered by a gasmined bed subsidence
}

\author{
Pieter C. Roos*, Suzanne J.M.H. Hulscher \\ Water Engineering and Management, Faculty of Engineering Technology, University of Twente, P.O. Box 217, 7500 AE, Enschede, \\ The Netherlands
}

Received 30 November 2000; accepted 11 January 2002

\begin{abstract}
Offshore gasmining is an example of a human intervention with a morphological impact. On land, it is usually attended with a dish-like bed depression. We show that, if located at sea, such a bed depression can become morphodynamically active by triggering mechanisms related to tidal sand bank formation. To that end, a simple morphological model is considered which describes an erodible bed subject to a tidal wave in a shallow sea. The continuous subsidence is modelled by a sink term in the sediment balance. Then, a linear approximation is carried out to describe the bed evolution after the onset of subsidence. The results, presented in physical space, show that the subsidence triggers the formation of a sand bank pattern that gradually spreads around the centre of subsidence, at a rate that may go up to $160 \mathrm{~m} \mathrm{year}^{-1}$, depending on the tidal transport rate and the tidal eccentricity. The dimension of the depression does not affect the spreading rate nor the orientation of the sand banks, but it does influence their spacing. The main conclusion is that the horizontal extent of the area influenced by the bed depression by far exceeds that of the direct subsidence, thus showing that bed depressions on land and at sea indeed behave in fundamentally different ways. The results suggest that nonlinear effects are worthwhile to be investigated in order to describe finite amplitude development of sand banks as well as the interaction between subsidence and bed forms.
\end{abstract}

(C) 2002 Elsevier Science Ltd. All rights reserved.

\section{Introduction}

Many shelf seas like the North Sea are used by man for a variety of purposes such as sand mining, navigation, gasmining and oil exploration. These activities are usually accompanied with interventions of morphologic impact, e.g. sand pits or deposits, construction and maintenance dredging of navigation channels and a subsiding bed due to gas drilling. Typical of these interventions is their spatially bounded character. In the present paper,

\footnotetext{
*Corresponding author.

E-mail address: p.c.roos@ctw.utwente.nl (P.C. Roos).
}

we focus on the latter type of human activity: submarine bed depressions due to gasmining. For bed depressions on land accurate models exist, taking into account the stresses in a inhomogeneous soil. For engineering purposes simple models can predict the main behaviour, see Houtenbosch (1998). At sea, however, an essentially different situation arises since a bed subsidence can become morphodynamically active, by triggering mechanisms related to the natural morphodynamics of the seabed.

The offshore seabed exhibits a wide variety of rhythmic bottom features of different length scales, such as sand banks, tidal ridges, sandwaves, 
megaripples and sand ripples. In the present paper, we restrict our attention to sand banks, which have a typical wave length of some kilometres and crest orientation slightly cyclonic (i.e. counterclockwise on the Northern Hemisphere) up to parallel with respect to the main tidal motion. Their formation can be explained as a morphodynamic instability of a sandy bed subject to horizontal tidal motion, see Huthnance (1982a), extended by De Vriend (1990), Hulscher et al. (1993) and Hulscher (1996). Each of these models treats the system as fully natural, i.e. without any isolated external forcings due to human intervention. In contrast to the human interventions and to observed tidal sand bank patches, the patterns emerging in these models have a spatially unbounded character. We note that natural disturbances are not necessarily spatially unbounded, i.e. they can also have a local character, such as earthquakes. The problem of tidal motion encountering local bed structures has been investigated by many authors, e.g. Loder (1980), Zimmerman (1981) and Yungshuo and Thompson (1997).

Huthnance (1982b) described the formation of sand banks of finite horizontal extent. He gives a detailed description of the evolution of elliptical sand banks in terms of longitudinal and lateral spreading, growth in height and volume, as well as rotation. However, restricting to the properties of an individual sand bank, Huthnance does not address the issue of pattern spreading. Moreover, the dependency of the results on the tidal eccentricity is left unattended.

In the present study we investigate sand bank formation triggered by a submarine bed depression due to gasmining, since the corresponding time and length scales suggest a strong relation. In doing so, we combine the work on tidal sand banks with that of the bed subsidence formulation of Fluit and Hulscher (2002) by including a continuous, dish-like bed depression to the morphodynamic part of the model. They showed that in case of a time-independent flow, the mechanisms indeed become active. Their conclusions are based on Fourier-transformed bed results, so they were unable to show the bed evolution in the physical space. This means that quantitative information on the spreading of depression and bank development could not be given. This information is vital in understanding the horizontal impact scale of the bed depression. Moreover, they considered stationary tidal flow. In this paper we will omit these shortcomings by

- transforming the results in Fourier space back into the physical space, which enables us to visualize both the hydrodynamic response to the bed depression and the resulting bed development; and

- considering more realistic flow conditions, an elliptical tide rather than stationary flow, and studying the dependency of the results on the tidal eccentricity, which varies considerably in the North Sea ranging from -0.6 (anticyclonic) to 0.6 (cyclonic), see Zongwan et al. (1995).

We allow the presence of initial bed forms, but most results are presented for an initially flat bed. Even though we know from the above that the basic state characterizing by a flat bed is essentially unstable, we feel that this is a sound way to start tackling the problem. In this way we study the morphodynamic response to the bed depression isolated from topography present due to inherent instability. Otherwise, we would have to specify the amplitude of the initial bed forms relative to the strength of the subsidence, which is meaningless in the linear context. As the model is linear in the amplitude of the bed perturbation, it is unable to describe the amplitude interaction between naturally forming sand banks and those forming in response to the subsidence. By definition, this would require a nonlinear approach which is, at present, not within reach.

The paper is organized as follows. Section 2 provides the theoretical framework, i.e. the formulation of the model combining tidal motion with bed subsidence. Section 3 presents the solution method. Then, after the numerical inverse Fourier transformation, the results in physical space are presented in Section 4. Finally, Section 5 contains the discussion and conclusions. 


\section{Model}

\subsection{Hydrodynamic part}

Consider a tidal wave with maximum velocity $U^{*}$, tidal frequency $\sigma^{*}$ in an offshore part of a shallow sea of undisturbed depth $H^{*}$. Unsteady flow can be described by the depth-averaged shallow-water equations, i.e. by two momentum equations and a mass balance. In dimensional form (an asterisk denotes a dimensional quantity), the model reads

$$
\begin{gathered}
g^{*} \frac{\partial \zeta^{*}}{\partial x^{*}}+\frac{\partial u^{*}}{\partial t^{*}}+u^{*} \frac{\partial u^{*}}{\partial x^{*}}+v^{*} \frac{\partial u^{*}}{\partial y^{*}} \\
-f^{*} v^{*}+\frac{r^{*} u^{*}}{H^{*}+\zeta^{*}-h^{*}}=0, \\
g^{*} \frac{\partial \zeta^{*}}{\partial y^{*}}+\frac{\partial v^{*}}{\partial t^{*}}+u^{*} \frac{\partial v^{*}}{\partial x^{*}}+v^{*} \frac{\partial v^{*}}{\partial y^{*}} \\
+f^{*} u^{*}+\frac{r^{*} v^{*}}{H^{*}+\zeta^{*}-h^{*}}=0, \\
\frac{\partial \zeta^{*}}{\partial t^{*}}-\frac{\partial h^{*}}{\partial t^{*}}+\frac{\partial}{\partial x^{*}}\left[\left(H^{*}+\zeta^{*}-h^{*}\right) u^{*}\right] \\
+\frac{\partial}{\partial y^{*}}\left[\left(H^{*}+\zeta^{*}-h^{*}\right) v^{*}\right]=0 .
\end{gathered}
$$

Here $\mathbf{u}^{*}=\left(u^{*}, v^{*}\right)$ are the velocity components in the directions of the horizontal coordinates $\mathbf{x}^{*}=\left(x^{*}, y^{*}\right)$, respectively. The $z^{*}$-axis points upward with the free surface elevation at $z^{*}=\zeta^{*}$ and the bed level at $z^{*}=-H^{*}+h^{*}$. Furthermore, $g^{*}$ is the gravitational acceleration. Effects of the earth's rotation are included with $f^{*}$ the Coriolis parameter. Finally, a linear friction law is incorporated with parameter $r^{*}$. The boundaries of the offshore system are assumed to be infinitely far away. See Fig. 1 for a definition sketch.

\subsection{Morphodynamic part}

The seabed is assumed to consist of cohesionless sediment of uniform size, which is transported mainly as bed-load. The bed-load transport can be modelled using a generalization of an empirical relationship including a slope correction, see, e.g. Van Rijn (1993). The bed

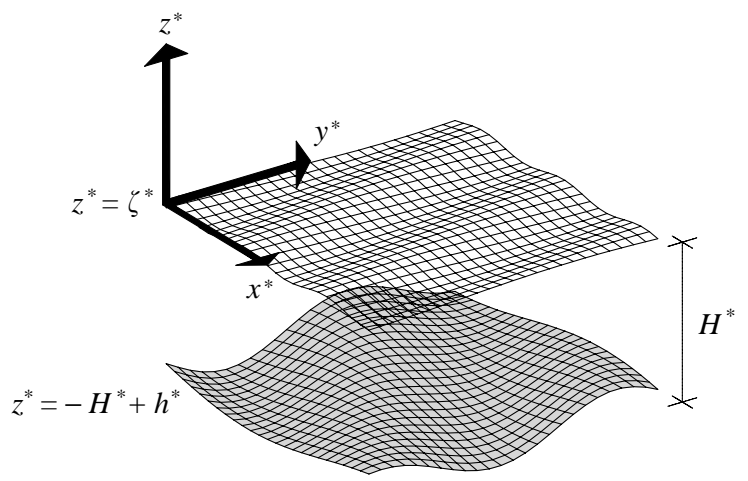

Fig. 1. Definition sketch of the model geometry.

development is described by

$\frac{\partial h^{*}}{\partial t^{*}}+\nabla^{*} \cdot\left[\alpha^{*}\left|\mathbf{u}^{*}\right|^{b}\left(\frac{\mathbf{u}^{*}}{\left|\mathbf{u}^{*}\right|}-\lambda^{*} \nabla^{*} h^{*}\right)\right]=-\rho^{*} h_{\mathrm{s}}$.

Here, sediment transport is characterized by three parameters: a proportionality parameter $\alpha^{*}$ that includes the effect of porosity, an exponent $b$ with a value usually between 3 and 5 reflecting the nonlinear character of sediment transport and a slope correction parameter $\lambda^{*}$ between 1 and 3, quantifying the downhill preference of moving sediment.

Moreover, Fluit and Hulscher (2002) have added a sediment sink term to Eq. (4) in order to account for the bottom subsidence, with a constant subsidence rate $\rho^{*}$ and a shape function $h_{\mathrm{s}}$. Using 10 years of data from the barrier island Ameland (West-Frisian Wadden Sea, The Netherlands), they show that the bed depression due to gas mining can be modelled as a dish-like Gaussian shape, i.e.

$h_{\mathrm{s}}\left(\mathbf{x}^{*}\right)=\mathrm{e}^{-(1 / 2)\left(x^{* 2}+y^{* 2}\right) / R^{* 2}}$.

Here, without loss of generality, the centre of subsidence is chosen to coincide with the origin of the coordinate system. The radius $R^{*}$, defined as the distance between the centre of the subsidence and its inflection circle, is shown to be constant with time. See Fig. 2 for a plot of the subsidence shape. Here we are mainly interested in the qualitative properties of the subsidence as well as the orders of magnitude. Parameter values typical 


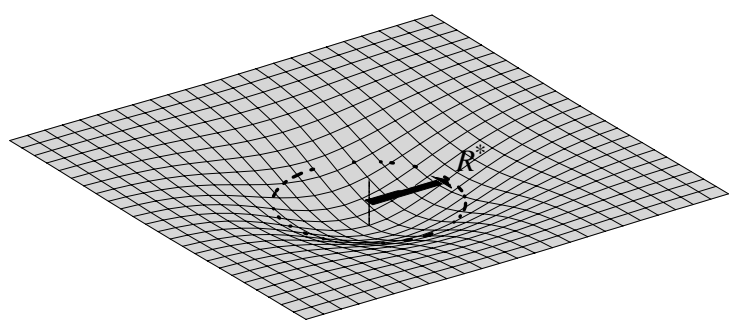

Fig. 2. Sketch of the subsidence shape and its inflection circle of radius $R^{*}$.

for the bed depression on Ameland are $\rho^{*}=$ $5.82 \times 10^{-10} \mathrm{~m} \mathrm{~s}^{-1}$ and $R^{*}=3.0 \mathrm{~km}$, which will be used hereafter. More details on the characteristics of the subsidence can be found in Fluit and Hulscher (2002).

Observe that the value of $R^{*}$ is of the same order of magnitude as the wavelength of sand banks. Finally, we note that the tidal sand bank model is recovered by taking $h_{\mathrm{s}} \equiv 0$.

\subsection{Scaling procedure}

The model is cast in nondimensional form by introducing the nondimensional variables

$$
\begin{aligned}
& \mathbf{u}=\frac{\mathbf{u}^{*}}{U^{*}}, \quad t=t^{*} \sigma^{*}, \quad \mathbf{x}=\frac{\sigma^{*}}{U^{*}} \mathbf{x}^{*}, \\
& h=\frac{h^{*}}{H^{*}}, \quad \zeta=\frac{g^{*}}{U^{* 2}} \zeta^{*} .
\end{aligned}
$$

We arrive at the following nondimensional model:

$$
\begin{aligned}
& \frac{\partial \zeta}{\partial x}+\frac{\partial u}{\partial t}+u \frac{\partial u}{\partial x}+v \frac{\partial u}{\partial y}-f v+\frac{r u}{1+\delta^{2} \zeta-h}=0, \\
& \frac{\partial \zeta}{\partial y}+\frac{\partial v}{\partial t}+u \frac{\partial v}{\partial x}+v \frac{\partial v}{\partial y}+f u+\frac{r v}{1+\delta^{2} \zeta-h}=0, \\
& \delta^{2} \frac{\partial \zeta}{\partial t}-\frac{\partial h}{\partial t}+\frac{\partial}{\partial x}\left[\left(1+\delta^{2} \zeta-h\right) u\right]+ \\
& \frac{\partial}{\partial y}\left[\left(1+\delta^{2} \zeta-h\right) v\right]=0,
\end{aligned}
$$$$
\frac{\partial h}{\partial t}+\alpha \nabla \cdot\left[|\mathbf{u}|^{b}\left(\frac{\mathbf{u}}{|\mathbf{u}|}-\lambda \nabla h\right)\right]=-\rho \mathrm{e}^{-\left(x^{2}+y^{2}\right) / L_{\mathrm{s}}^{2}} .
$$

Here, the following nondimensional parameters appear:

$$
\begin{aligned}
& \delta=\frac{U^{*}}{\sqrt{g^{*} H^{*}}}, \quad r=\frac{r^{*}}{\sigma^{*} H^{*}}, \quad f=\frac{f^{*}}{\sigma^{*}}, \quad \alpha=\frac{U^{* b-1}}{H^{*}} \alpha^{*}, \\
& \lambda=\frac{H^{*} \sigma^{*}}{U^{*}} \lambda^{*}, \quad \rho=\frac{\rho^{*}}{H^{*} \sigma^{*}}, \quad L_{\mathrm{s}}=\frac{\sqrt{2} R^{*} \sigma^{*}}{U^{*}} .
\end{aligned}
$$

Now we will discuss values of the parameters involved, typical for the North Sea. The maximum tidal velocity $U^{*}$ is of the order of $1 \mathrm{~m} \mathrm{~s}^{-1}$. The frequency of the $M_{2}$-tide in the North Sea is $1.4 \times$ $10^{-4} \mathrm{~s}^{-1}$. At $52^{\circ} \mathrm{N}$ the value of the Coriolis parameter is given by $f^{*}=1.16 \times 10^{-4} \mathrm{~s}^{-1}$. The friction parameter $r^{*}$ can be estimated from the drag coefficient. Following Hulscher et al. (1993), for a typical depth of about $30 \mathrm{~m}$, we take a friction parameter $r$ from the range between 0.2 and 2.4; we choose a value of $r=0.6$. The constant $\alpha^{*}$ can be computed, e.g. from van Rijn (1993), which leads to a value used by Hulscher et al. (1993) of $1.5 \times 10^{-5} \mathrm{~m}^{2-b} \mathrm{~s}^{b-1}$. However, wave effects are not included here and thus we expect that the actual value of $\alpha^{*}$ will be higher. Huthnance (1982a), e.g. used a significantly higher value of $2.7 \times 10^{-4} \mathrm{~m}^{2-b} \mathrm{~s}^{b-1}$. The uncertainty in estimating $\alpha^{*}$ creates uncertainty in the morphological time scales. Therefore we will formulate the results for a range of $\alpha^{*}$ for which we choose $1.5 \times 10^{-5}-1.5 \cdot 10^{-4} \mathrm{~m}^{2-b} \mathrm{~s}^{b-1}$ in which values associated with the largest transport are denoted by italics throughout this paper. For the other sediment transport parameters we take $b=3$ and $\lambda^{*}=2$. Values of the bed depression parameters are taken from Section 2.2. The numerical values of the corresponding dimensionless parameters (11) are given in Table 1 . The fact that the parameter $L_{\mathrm{s}}$ is of order one shows that the dimension of the bed depression and the topographic length scale (tidal excursion) are of the same order of magnitude.

\subsection{Time scales, averaging and initial topography}

The parameters $\alpha^{*}$ and $\rho^{*}$ in Eq. (10) reflect the importance of the two morphological effects, i.e. bed evolution due to the natural morphodynamics and the subsiding bed, respectively. The 
Table 1

Numerical values of the nondimensional model parameters defined in Eq. (11) for a typical North Sea situation

\begin{tabular}{|c|c|c|c|c|c|c|c|c|}
\hline \multirow{2}{*}{$\frac{\text { Category }}{\text { Parameter }}$} & \multicolumn{3}{|c|}{ Hydrodynamics } & \multicolumn{3}{|c|}{ Sediment transport } & \multicolumn{2}{|c|}{ Bed depression } \\
\hline & $\delta$ & $r$ & $f$ & $\alpha$ & $b$ & $\lambda$ & $\rho$ & $L_{\mathrm{s}}$ \\
\hline Value & 0.06 & 0.6 & 0.83 & $\begin{array}{l}0.5 \times 10^{-6} \\
0.5 \times 10^{-5}\end{array}$ & 3 & $8.4 \times 10^{-3}$ & $1.39 \times 10^{-7}$ & 0.60 \\
\hline
\end{tabular}

morphological time scale $T_{\mathrm{m}}$, being the e-folding time of sand banks, takes a value of $T_{\mathrm{m}}=\left(\alpha \sigma^{*}\right)^{-1}=45-450$ years, emphasizing the effect of uncertainty in $\alpha^{*}$ on the morphological time scale. Huthnance (1982a) initially found a time scale of only 20 years, which he corrected to 100 years based on his results. The idea of taking both fair weather and storms into account has been applied in detail by Calvete et al. (2001) to the case of shoreface-connected ridges. They found that the morphological time scales of stormdominated shelves and shelves characterized by fair weather conditions may differ by one order of magnitude. Based on the continuous subsidence of about $2 \mathrm{~cm} \mathrm{year}^{-1}$ we find a subsidence time scale of $T_{\mathrm{s}}=\left(\rho \sigma^{*}\right)^{-1}=1600$ years, defined as the time it takes for the subsidence to equal the full water depth, here $30 \mathrm{~m}$.

The fact that both morphological effects take place on time scales much larger than that of the tide justifies the decoupling of the hydrodynamic and the morphodynamic part. This is a common procedure (see, e.g. De Vriend, 1990; Hulscher et al., 1993; and Calvete et al., 2001), that involves introducing the slow time scale $\tau=\alpha t$ and dropping $\partial h / \partial t$ in Eq. (9). Hence we study the bed development on the time scale of the natural morphodynamics $T_{\mathrm{m}}$. The bottom evolution equation (10) in terms of the slow time $\tau$ reads

$\frac{\partial h}{\partial \tau}+\nabla \cdot\left\langle|\mathbf{u}|^{b}\left(\frac{\mathbf{u}}{|\mathbf{u}|}-\lambda \nabla h\right)\right\rangle=-\mu h_{\mathrm{s}}$,

where $\langle\cdot\rangle$ denotes averaging over a tidal cycle. From the differences in time scales it is immediately seen that the sink term is relatively small and of order $\mu=\rho / \alpha=T_{\mathrm{m}} / T_{\mathrm{s}}=0.028-0.28$. Regarding the solution procedure, the decoupling means that first the hydrodynamic equations (7)-(9) are solved with $\partial h / \partial t=0$, independent of the morphodynamic equation (12) which is solved next.
Finally, it is important that initial topographic perturbations are either absent or at most of order $\mu$ :

$\left.h(x, y)\right|_{\tau=0}=\mu h_{\text {init }}(x, y)$.

In the absence of bottom subsidence, the parameter $\mu$ in Eq. (13) should be interpreted as a strictly infinitesimal expansion parameter rather than a finite ratio of two time scales.

\section{Solution method}

\subsection{Linear approximation concept}

The solution of the problem, symbolically written as the vector $\phi=(u, v, \zeta, h)$, cannot be found in exact form. Therefore, the idea is to perform a linear approximation in the small parameter $\mu$ :

$\phi=\phi_{0}+\mu \phi_{1}+\mathcal{O}\left(\mu^{2}\right)$.

Inserting Eq. (14) into the model equations and collecting like powers of $\mu$ yields problems at different orders of approximation. We neglect terms proportional to $\delta^{2}$ since $\delta^{2} \ll \mu \ll 1$. At the lowest order of approximation, neither the initial topography nor the bottom subsidence is present and we impose an elliptical tide of the form

$\phi_{0}=\left(u_{0}, v_{0}, \zeta_{0}, h_{0}\right)=\left(\cos t, \varepsilon \sin t, \zeta_{0}(x, y, t), 0\right)$.

The tidal eccentricity parameter $\varepsilon$ permits the modelling of both cyclonic (counterclockwise, $\varepsilon>0$ ) and anticyclonic (clockwise, $\varepsilon<0$ ) tides. ${ }^{1}$

\footnotetext{
${ }^{1}$ This holds on the Northern Hemisphere. On the Southern Hemisphere the opposite is true: cyclonic means clockwise and anticyclonic counterclockwise.
} 
Unidirectional oscillatory tides and circular tides correspond to $\varepsilon=0$ and \pm 1 , respectively. At order $\mu$ a set of equations, linear in $\phi_{1}$, is found (see Appendix A).

\subsection{Solving the linear problem}

The momentum equations of the linear problem can easily be formulated in terms of the vorticity $\eta=\partial v / \partial x-\partial u / \partial y$, by cross-differentiating and subtracting the momentum equations (A.1) and (A.2). This gives, with the use of the continuity equation (A.3) in order to eliminate $u$ and $v$, an equation in terms of $\eta_{1}$ and $h_{1}$ only. This equation will be solved in Fourier space, so we define Fourier variables according to

$\eta_{1}(\mathbf{x}, t)=\iint \hat{\eta}_{1}(\mathbf{k}, t) \mathrm{e}^{-i \mathbf{k} \cdot \mathbf{x}} \mathrm{d} \mathbf{k}+$ c.c.

with wave vector $\mathbf{k}=(k, \ell)$ and $\hat{h}_{1}$ is defined analogously. The vorticity equation in Fourier space reads

$$
\begin{aligned}
\frac{\partial \hat{\eta}_{1}}{\partial t}= & (\mathrm{i} k \cos t+\mathrm{i} \ell \varepsilon \sin t-r) \hat{\eta}_{1} \\
& +[\mathrm{i}(k f-\ell r) \cos t \\
& +\mathrm{i}(k r+\ell f) \varepsilon \sin t] \hat{h}_{1} .
\end{aligned}
$$

Interaction between $\hat{\eta}_{1}$ and the lowest order flow solution (15) generates vorticity in all overtones of the basic tidal frequency. The solution procedure for the vorticity is analogous to the one described in Hulscher et al. (1993). Once Fourier transformed, the bottom evolution equation (A.4) can be expressed in terms of $\hat{h}_{1}$ :

$\frac{\partial \hat{h}_{1}}{\partial \tau}=\omega \hat{h}_{1}-\hat{h}_{\mathrm{s}}, \quad \omega=\omega(\mathbf{k} ; \varepsilon, b, f, r)$.

The full expression for $\omega$ is given in Eq. (A.5). If the subsidence were absent, $\omega$ would have the usual meaning of a growth rate. Essential here is that $\omega$ does not depend on the characteristics of the bed subsidence. Due to the symmetry of the problem, $\omega$ has a vanishing imaginary part, which means that bed forms do not migrate over a tidal cycle. Fig. 3 shows contour plots of the growth rate $\omega$ for various values of the tidal eccentricity $\varepsilon$. The Fourier transformed counterpart of the Gaussian
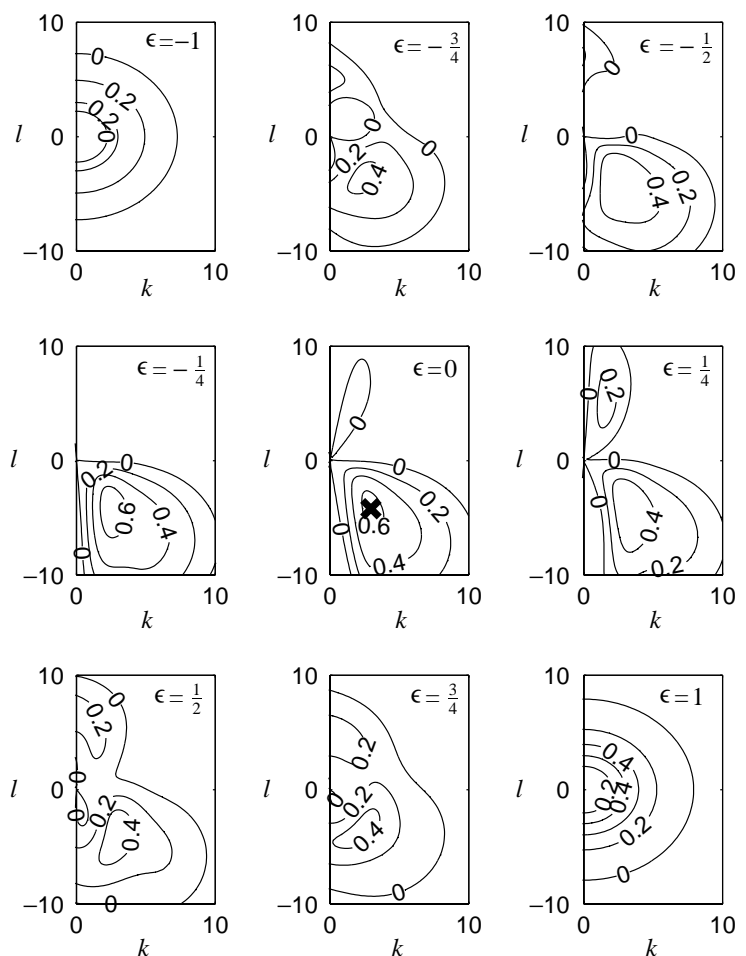

Fig. 3. Contour plots of the growth rate $\omega$ in the Fourier space for different values of the tidal eccentricity $\varepsilon$, ranging from -1 to 1 , as specified in each of the individual plots. Only nonnegative contours are plotted. Other parameter values are taken from Table 1. The cross in the centre plot refers to the fastest growing mode for $\varepsilon=0$, which will serve as a reference case in Fig. 6.

subsidence shape function (5) reads

$\hat{h}_{\mathrm{s}}=\frac{L_{\mathrm{s}}^{2}}{4 \pi} \mathrm{e}^{-(1 / 4) L_{\mathrm{s}}^{2}\left(k^{2}+\ell^{2}\right)}$.

Solving the bottom evolution equation (18) gives

$\hat{h}_{1}(\tau)=\hat{h}_{\text {init }} \mathrm{e}^{\omega \tau}-\hat{h}_{\mathrm{s}} \frac{\mathrm{e}^{\omega \tau}-1}{\omega}$,

where $\hat{h}_{\text {init }}$ is the Fourier transform of the initial topography (13). The above equation shows that the theory leading to the growth rate $\omega$ is unaffected by the bed subsidence process. Hence, from a computational perspective, only in the final stage of the morphodynamic part, in Eq. (18), the bottom subsidence $h_{\mathrm{s}}$ explicitly comes into play. 
In order to visualize the hydrodynamic response to the bed depression (see Fig. 4 in Section 4.1) we introduce a stream function $\psi$ according to

$(1-h) u=\frac{\partial \psi}{\partial y}, \quad(1-h) v=-\frac{\partial \psi}{\partial x}$.

This way, the continuity equation (9) (in which we neglected $\partial h / \partial t$ and terms of order $\delta^{2}$ ) is automatically satisfied. Similar to Eq. (14) we expand $\psi$ in powers of $\mu$. Even though $\psi$ defines discharge contours, plotting contours of the tidal average $\left\langle\psi_{1}\right\rangle$ shows the residual currents:

$\frac{\partial\left\langle\psi_{1}\right\rangle}{\partial y}=\left\langle u_{1}-h_{1} u_{0}\right\rangle=\left\langle u_{1}\right\rangle$

$\frac{\partial\left\langle\psi_{1}\right\rangle}{\partial x}=-\left\langle v_{1}-h_{1} v_{0}\right\rangle=-\left\langle v_{1}\right\rangle$.

\subsection{Analysing the bottom evolution equation}

The bottom development (20) can alternatively be formulated as

$$
\hat{h}_{1}(\tau)=\underbrace{\hat{h}_{\text {init }} \mathrm{e}^{\omega \tau}}_{\text {(i) }}-\underbrace{\hat{h}_{\mathrm{s}} \tau}_{\text {(ii) }}-\underbrace{\hat{h}_{\mathrm{s}}\left(\frac{\mathrm{e}^{\omega \tau \tau}-1}{\omega}-\tau\right)}_{\text {(iii) }} .
$$

Here three terms can be identified: (i) the natural morphodynamic behaviour, describing the exponential growth of initially present topographic perturbations $\hat{h}_{\text {init }}$, (ii) the direct bottom subsidence as it would develop on land and (iii) an additionally triggered term due to the simultaneous activity of both natural morphodynamics and bottom subsidence. The ratio of (iii) and (ii) gives an impression of the relative importance of this additional term, i.e. a comparison between land and sea. It can be developed in a Taylor series according to

$\frac{\mathrm{e}^{\omega \tau}-1}{\omega \tau}-1=\frac{1}{2} \omega \tau+\frac{1}{6} \omega^{2} \tau^{2}+\cdots$,

clearly showing that, as time evolves, term (iii) becomes increasingly important with respect to the direct subsidence term. Of course the linear character of this analysis sets a limit to the applicability of the theory for large $\tau$. By taking $\hat{h}_{\mathrm{s}}=0$ the original results from a stability analysis are recovered. Note also that when $\omega \downarrow 0$, we recover the bed subsidence on land.
At this point, another comparison may seem appropriate: the importance of the natural morphodynamics (i) relative to the morphodynamics (ii) and (iii) triggered by the subsidence. However, in the present linear approach, this implies a discussion on the ratio of $\hat{h}_{\mathrm{s}}$ and $\hat{h}_{\text {init }}$. Such information cannot be estimated directly from nature and we feel that this comparison should be done in a nonlinear context, in which $\hat{h}$ is not restricted to small values. That is why in the following we choose $\hat{h}_{\text {init }} \equiv 0$, effectively studying the case $\hat{h}_{\mathrm{s}} \gg \hat{h}_{\text {init }}$.

\section{Results in the physical space}

\subsection{Hydrodynamic response and bed development}

The expression for the bottom evolution (20) is available in Fourier space. In order to transform back to the physical space we numerically integrate Eq. (16) using standard techniques (see, e.g. Canuto et al., 1988). Next, we discuss the results for a unidirectional oscillatory tide $(\varepsilon=0)$ for a typical offshore North Sea situation (parameters as in Table 1). The results are depicted in Fig. 4.

At $\tau=0$ the bed is flat $\left(h_{\text {init }} \equiv 0\right)$ and there are no residual currents. However, immediately after the onset of the bed depression, horizontal cells appear around the centre of subsidence and a bed form pattern gradually appears. It starts as four humps and four pits surrounding the subsidence centre. The humps merge two-by-two, while two of the pits merge with the original subsidence. As a result, we see two elongated ridges flanking the original subsidence pit, which also has taken an elongated form. As time evolves, the patch of sand banks spreads, alternatingly adding troughs and crests, with the existing sand banks elongating further. The interaction between hydrodynamics and morphodynamics is evident. Flow convergence and divergence lead to bed rise and bed sink, respectively, and bed changes in turn modify the recirculating cell pattern. We note that residual cells are cyclonic (counterclockwise) around troughs and anticyclonic (clockwise) around crests, which agrees with the findings by Zimmerman (1981) and Huthnance (1982b). 

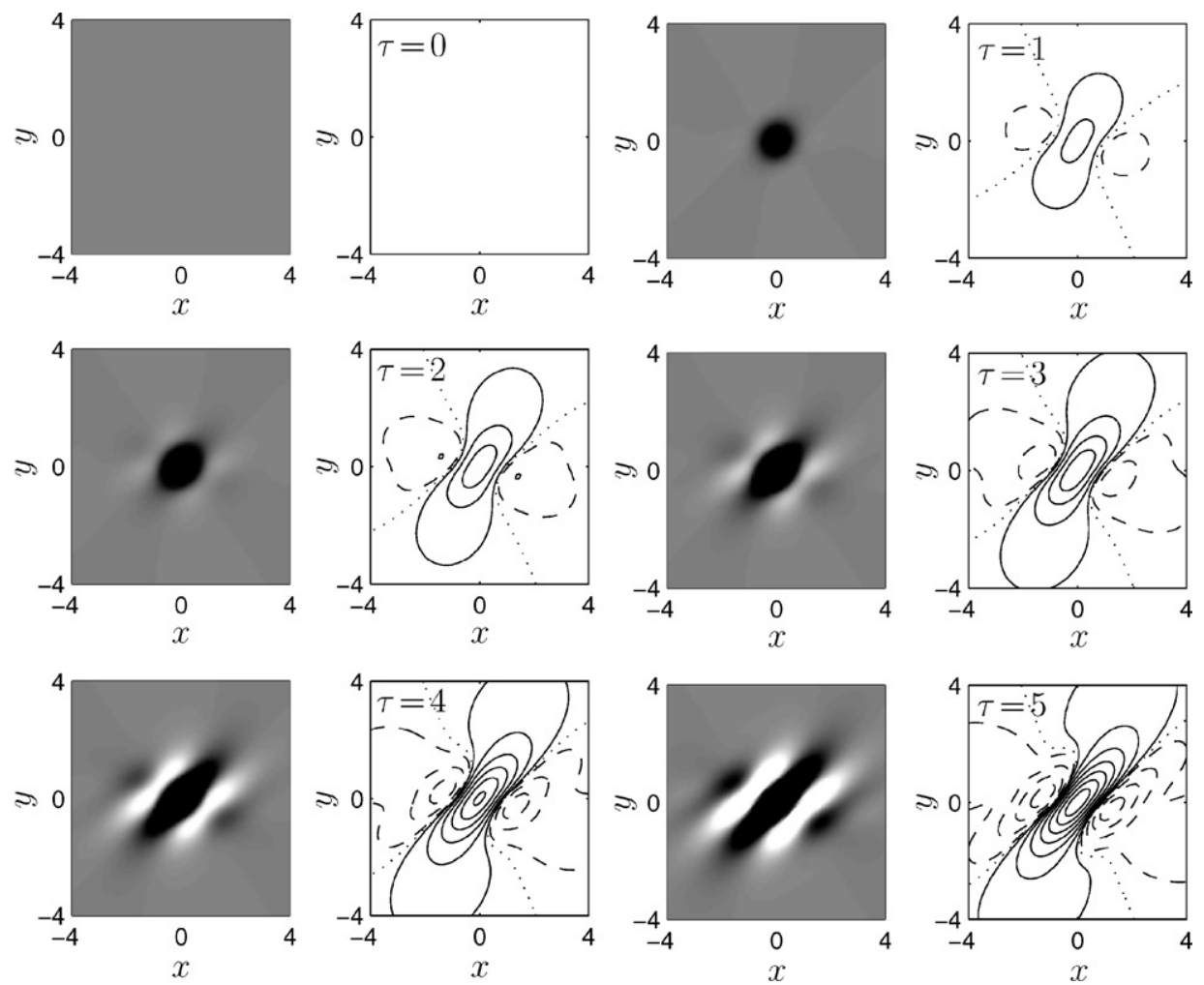

Fig. 4. Time evolution of both seabed and residual currents, from $\tau=0$ (top left) to $\tau=5$ (bottom right). Crests are white, troughs black and the undisturbed bed is grey. Contours of $\left\langle\psi_{1}\right\rangle$ are plotted, as defined in Eq. (22), with solid contours denoting counterclockwise circulation and dashed contours denoting clockwise circulation. A unidirectional oscillatory tide in $x$-direction is considered $(\varepsilon=0)$ and initially no topographic perturbations are present: $h_{\text {init }} \equiv 0$. For other parameter values see Table 1 . The plotted domain covers a dimensional area of about $56 \times 56 \mathrm{~km}^{2}$.

Fig. 5 shows the dependency of the bed topography at $\tau=5$ on the tidal eccentricity $\varepsilon$. Irrespective of the value of $\varepsilon$, a sand bank pattern emerges. The patterns for cyclonic tides and anticyclonic tides exhibit notable differences, also with respect to the degree of spreading. Finally, observe that both in the anticyclonically circular $(\varepsilon=-1)$ and in the cyclonically circular case $(\varepsilon=1)$, the pattern obeys rotational symmetry, as can be expected.

We note that $\tau=5$ may be beyond the applicability limit of this model. However, here we wish to show the tendency of the bed behaviour as predicted by the model. Moreover, in cases with very small $\mu$-values, the linear model is likely to hold for a longer period of time.

\subsection{Further analysing the radiation of the pattern}

The orientation of the features with respect to the tidal motion as well as their wavelength turn out to depend on the characteristics of both hydrodynamics and bed depression.

The pattern spreading is much more profound than the direct horizontal impact of the bed depression itself. This is mainly due to the fact that the bottom subsidence itself remains rather local. In order to quantify this concept, we define the pattern border as the most outward points where $h_{1}=h_{\text {imp }}$ and we choose $h_{\text {imp }}=0.05$. This way the pattern border is defined by $h=\mu h_{\text {imp }}$ and the radiation rate will not depend on $\mu$. We avoid an absolute criterion since it would result in 

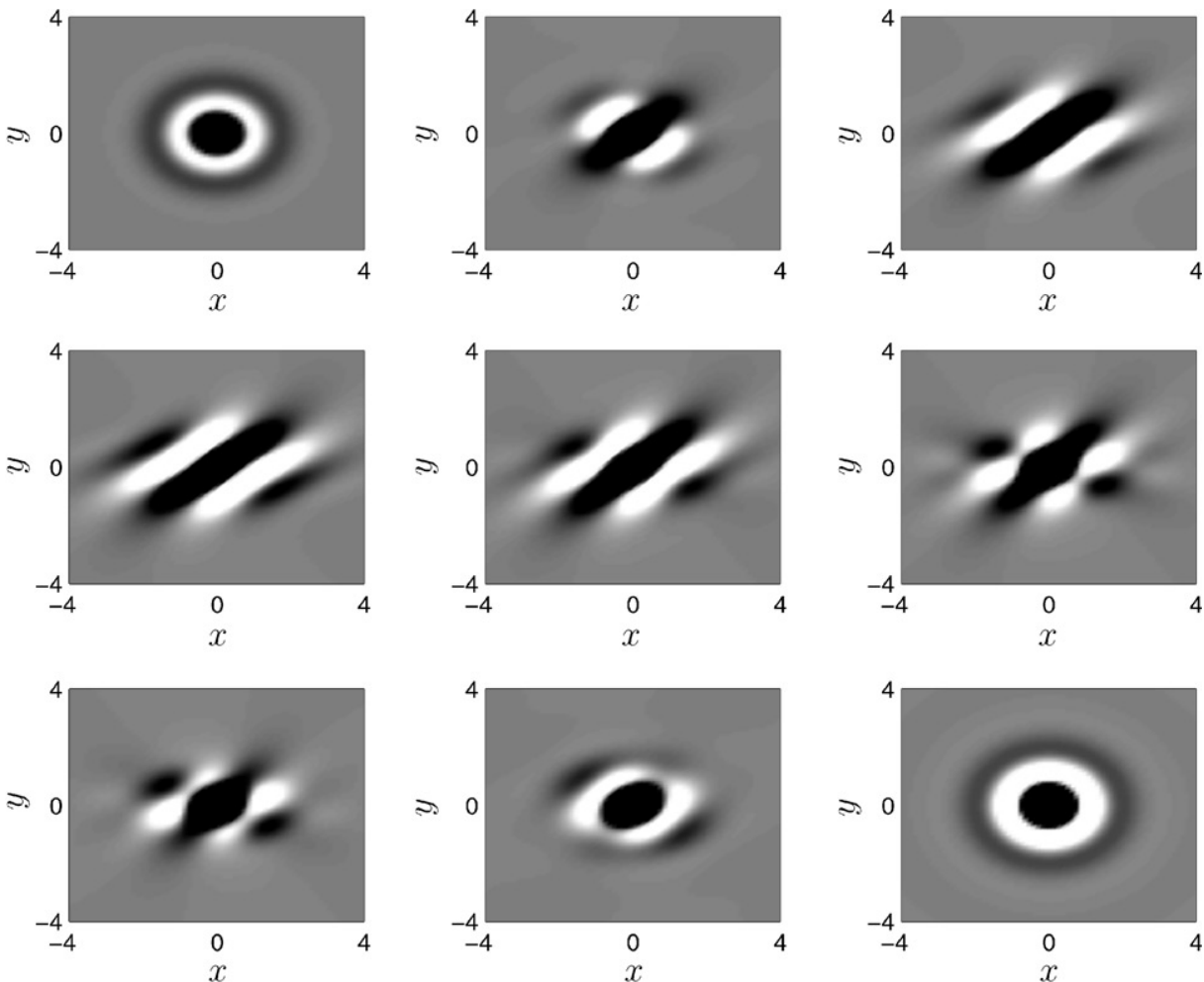

Fig. 5. Bed topography at $\tau=5$ for the values of tidal eccentricity introduced in Fig. 3. Initially no bed forms are present: $h_{\text {init }} \equiv 0$. The plotted domain covers a dimensional area of about $56 \times 56 \mathrm{~km}^{2}$.

radiation rates depending on $\mu$. Isolating the bottom subsidence, i.e. considering only term (ii) in Eq. (23), we find that the border is a circle of radius:

$$
\frac{R_{\mathrm{imp}}^{*}(\tau)}{R^{*}}=\sqrt{2 \ln \frac{\tau}{h_{\mathrm{imp}}}} .
$$

Hence, the bed depression itself spreads rather slowly. Without morphodynamic influences, it would take about 1000-10,000 years for the distance from pattern border to subsidence centre to increase by a factor of only 3.5.

Now switching on the morphodynamic activity (and taking $\varepsilon=0$ ), we study the morphologic pattern spreading by following the length of the central trough $L_{\mathrm{tr}}^{*}$ in time, restricting ourselves to term (iii) in Eq. (23). Contrary to the bed depression, it is found that the central trough elongates at a constant rate of about $16-160$ m year $^{-1}$, see Fig. 6. This growth is much faster than that of
Eq. (25) since, e.g. the spreading factor of 3.5 mentioned above is already attained after $90-900$ years. We note that some of the lines in plot (i) of Fig. 6 are not given for small $\tau$-values as $h_{\text {imp }}$ is not yet attained there.

We observe no dependency of the elongation rate on $R^{*}$ as all curves in Fig. 6 are of equal slope. For increasing $R^{*}$ the curves shift upward, which is simply due to the bed depression covering a larger horizontal area. The dependency of the sand bank spacing on the bed depression radius $R^{*}$ is more apparent. A bed depression of large diameter promotes the growth of sand banks with large wavelengths, see Fig. 6. Again, we allow large $\tau$ values to show the tendency of the model. Apparently, the wavelength of the features is proportional to the dimensions of the bed subsidence. This is because the bed subsidence is modelled as a continuous sink, so that its spatial characteristics are continuously fed to the system. 

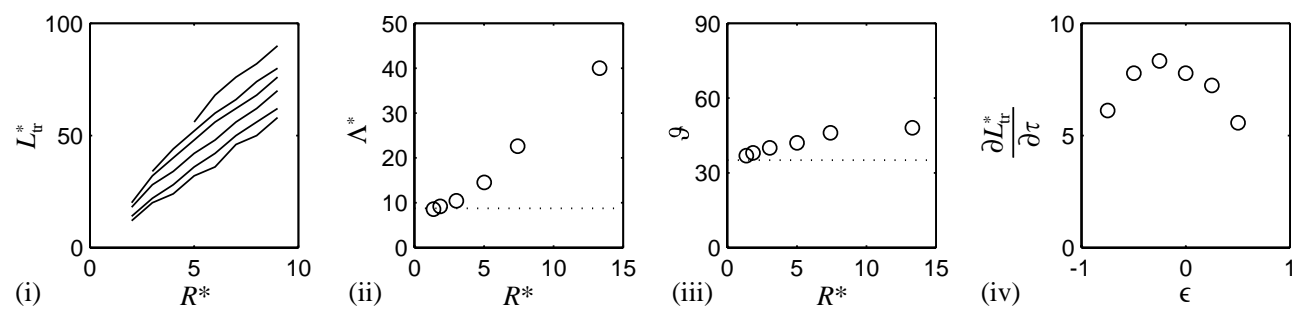

Fig. 6. Properties of the pattern spreading. (i) Central trough length $L_{\mathrm{tr}}^{*}$ (in km) versus time (in units of $\tau$ ) for various values of $R^{*}$ (bottom to top): 1.4, 1.9, 3.0, 5.0, 7.4, $13.3 \mathrm{~km}$. (ii) Wavelength $\Lambda^{*}$ of the sand banks (in $\mathrm{km}$ ) as a function of $R^{*}$ (in $\mathrm{km}$ ). (iii) Angle $\vartheta$ between central trough and direction of the tidal motion as a function of $R^{*}$ (in $\mathrm{km}$ ). Wavelength and angle as predicted for an undisturbed environment, i.e. without subsidence, are denoted by a dotted line. These correspond to the cross in the centre plot of Fig. 3 . Tidal eccentricity taken zero. (iv) Dependency of elongation rate of central trough $\frac{\partial L_{\mathrm{tr}}^{*}}{\partial \tau}$ (in $\mathrm{km}$ per unit of $\tau$ ) on tidal eccentricity $\varepsilon$.

Fig. 6 also shows that the orientation of the sand banks with respect to the main tidal motion hardly depends on $R^{*}$. Finally, we see that the elongation rate strongly depends on the value of the tidal eccentricity. The pattern spreads fastest for $\varepsilon$ just smaller than zero, i.e. for slightly anticyclonic (i.e. clockwise on the Northern Hemisphere) tides. Moreover, we note that for $\varepsilon$ close to \pm 1 , the elongation rate of the central trough is no measure for the pattern spreading, as the central trough does not elongate at all in these rotationally symmetric cases (see also Fig. 5).

\section{Discussion and conclusions}

Conceptually the theories by Huthnance (1982a) and Hulscher et al. (1993) on the one hand and Fluit and Hulscher (2002) as well as this paper on the other hand exhibit some fundamental differences. The former are a classical linear stability analysis where the presence of initial topographic perturbations is a prerequisite for morphological activity. Moreover, the expansion parameter $\mu$ is strictly assumed to be infinitesimally small. For the latter models, the continuous bottom subsidence provides a mechanism that automatically triggers morphological development. Hence, an initial topograhpy not required to initiate bed development. Moreover, $\mu$ is the finite ratio of the morphological time scale $T_{\mathrm{m}}$ and the forcing time scale $T_{\mathrm{s}}$. Rather than a stability analysis, this theory is a linear approximation of the bed development after the bed subsidence has started. Hence, the moment $\tau=0$ has a clear significance: it is the onset of the subsidence. The model with subsidence has a local character where bed forms will develop and spread around the centre of subsidence, whereas an offshore stability analysis is usually employed to describe the formation of bed forms on an infinite horizontal domain.

The applicability of the results is of course limited to small values of $\tau$ as the linear theory predicts uninhibited growth of bed forms. This would result in very high crests, deep troughs and, hence, very steep bed forms, especially close to the subsidence centre. Therefore, the present theory provides an indication of the spreading pattern for small values of $\tau$. Nonlinear theory should be applied to investigate both amplitude development and pattern spreading when amplitudes are no longer small. This also requires insight into the nonlinear behaviour of sand banks in an undisturbed environment, i.e. without any bed depression.

Moreover, experimental verification is difficult. Nevertheless, the main conclusion of the present study is that a local intervention has a morphological impact far beyond the area of intervention and that the horizontal impact spreads at a rate of about $16-160 \mathrm{~m}$ year $^{-1}$. Similar behaviour can be expected for sand mining pits in a sand wave area. Sand waves develop on shorter time scales (typically 1-10 years) so that results of pattern spreading due to human intervention for that application could be tested against data within a reasonable time. 
It has been shown that submarine bed depressions at sea behave in a completely different way than bed depressions on land. A pattern of sand banks gradually appears and spreads around the centre of subsidence, showing that the horizontal impact scale extends beyond that of the direct subsidence. The pattern itself as well as the spreading rate depend on the tidal eccentricity with fastest spreading predicted for $\varepsilon$ just below zero, i.e. for anticyclonic tides of small eccentricity. The findings concerning the pattern (shape, spreading celerity, $\varepsilon$-dependence) constitute an extension to the results by Fluit and Hulscher (2002).

The uninhibited growth predicted by a linear analysis suggests it is worthwhile to investigate amplitude development and pattern spreading also in a nonlinear way. Moreover, the implication of including storm events on the value of the sediment transport factor $\alpha^{*}$ deserves more attention, due to its impact on the dimensional value of the morphological time scale and on the spreading rate.

\section{Acknowledgements}

The authors thank Huib de Vriend, for his useful comments on an earlier version of the manuscript. This work has been carried out within the framework of the EU-project HUMOR, contract number EVK3-CT-2000-00037.

\section{Appendix A. Model equations}

The linear problem, obtained at order $\mu$ in Section 3, reads

$$
\begin{gathered}
\frac{\partial \zeta_{1}}{\partial x}+\frac{\partial u_{1}}{\partial t}+u_{0} \frac{\partial u_{1}}{\partial x}+v_{0} \frac{\partial u_{1}}{\partial y} \\
-f v_{1}+r\left(h_{1} u_{0}+u_{1}\right)=0 \\
\frac{\partial \zeta_{1}}{\partial y}+\frac{\partial v_{1}}{\partial t}+u_{0} \frac{\partial v_{1}}{\partial x}+v_{0} \frac{\partial v_{1}}{\partial y} \\
+f u_{1}+r\left(h_{1} v_{0}+v_{1}\right)=0
\end{gathered}
$$

$$
\begin{aligned}
& \frac{\partial u_{1}}{\partial x}+\frac{\partial v_{1}}{\partial y}-u_{0} \frac{\partial h_{1}}{\partial x}-v_{0} \frac{\partial h_{1}}{\partial y}=0 \\
& \frac{\partial h_{1}}{\partial \tau}+\nabla \cdot\left\langle\left|\mathbf{u}_{0}\right|^{b-1} \mathbf{u}_{1}+(b-1)\left|\mathbf{u}_{0}\right|^{b-3} \mathbf{u}_{0}\left(\mathbf{u}_{0} \times \mathbf{u}_{1}\right)\right. \\
& \left.\quad-\left|\mathbf{u}_{0}\right|^{b} \lambda \nabla h_{1}\right\rangle=-\mathrm{e}^{-|\mathbf{x}|^{2} / L_{\mathrm{s}}^{2}}
\end{aligned}
$$

The expression for the growth rate $\omega$ reads

$$
\begin{aligned}
\omega= & -\lambda\left(k^{2}+\ell^{2}\right)\left\langle\left(\cos ^{2} t+\varepsilon^{2} \sin ^{2} t\right)^{b / 2}\right\rangle \\
& +\frac{b-1}{k^{2}+\ell^{2}}\left\langle\left[\frac{1}{2}\left(k^{2}-\ell^{2}\right) \sin 2 t\right.\right. \\
& \left.+k \ell\left(\varepsilon^{2} \sin ^{2} t-\cos ^{2} t\right)\right] \\
& \left(\cos ^{2} t+\varepsilon^{2} \sin ^{2} t\right)^{(b-3) / 2}\left[d_{0}\right. \\
& \left.\left.+\sum\left(d_{\mathrm{s} p} \sin p t+d_{\mathrm{cp}} \cos p t\right)\right]\right\rangle .
\end{aligned}
$$

Only in the special cases of a unidirectional oscillatory tide $(\varepsilon=0)$ or a circular tide $(\varepsilon= \pm 1)$ this expression can be simplified, see Hulscher et al. (1993).

\section{References}

Calvete, D., Walgreen, M., De Swart, H.E., Falqués, A., 2001. A model for sand ridges on the shelf: effect of tidal and steady currents. Journal of Geophysical Research 106 (C5), 9311-9338.

Canuto, C., Hussaini, M.Y., Quarteroni, A., Zang, T.A., 1988. Spectral Methods in Fluid Dynamics. Springer, New York.

De Vriend, H.J., 1990. Morphological processes in shallow tidal seas. In: Cheng, R.T. (Ed.), Residual Currents and Long Term Transport, Coastal and Estuarine Studies, Vol. 38. Springer, Berlin, pp. 276-301.

Fluit, C.C.J.M., Hulscher, S.J.M.H., 2002. Morphological response to a North seabed depression induced by gas mining. Journal of Geophysical Research 107, 10.1029/ 2001JC000851.

Houtenbosch, A.P.E.M., 1998. Bottom depression Ameland 1986-1998. Report 1998 03000426, NAM Topographical Department, Assen (in Dutch).

Hulscher, S.M.J.H., De Swart, H.E., De Vriend, H.J., 1993. The generation of offshore tidal sand banks and sand waves. Continental Shelf Research 13 (11), 1183-1204.

Hulscher, S.J.M.H., 1996. Tidal-induced large-scale regular bed form patterns in a three-dimensional shallow water model. Journal of Geophysical Research 101, 20,727-20,744.

Huthnance, J., 1982a. On one mechanism forming linear sand banks. Estuarine and Coastal and Shelf Science 14, 79-99.

Huthnance, J., 1982b. On the formation of sand banks of finite extent. Estuarine and Coastal Shelf Science 15, 277-299. 
Loder, J.W., 1980. Topographic rectification of tidal currents on the sides of Georges Bank. Journal of Physical Oceanography 10, 1399-1416.

Van Rijn, L.C., 1993. Handbook of Sediment Transport by Currents and Waves. Delft Hydraulics, Delft, The Netherlands. Yungshuo, S., Thompson, K.R., 1997. Oscillating flow of a homogenous fluid over an isolated topographic feature. Atmosphere-Ocean 35 (3), 229-255.
Zimmerman, J.T.F., 1981. Dynamics, diffusion and geomorphological significance of tidal residual eddies. Nature 290, $549-555$.

Zongwan, X., Carbajal, N., Sündermann, J., 1995. Tidal current amphidromic system in semi-enclosed basins. Continental Shelf Research 15 (2/3), 219-240. 